\title{
Digital Waveguides versus Finite Difference Structures: Equivalence and Mixed Modeling
}

\author{
Matti Karjalainen \\ Laboratory of Acoustics and Audio Signal Processing, Helsinki University of Technology, 02150 Espoo, Finland \\ Email: matti.karjalainen@hut.fi \\ Cumhur Erkut \\ Laboratory of Acoustics and Audio Signal Processing, Helsinki University of Technology, 02150 Espoo, Finland \\ Email: cumhur.erkut@hut.fi
}

Received 30 June 2003; Revised 4 December 2003

\begin{abstract}
Digital waveguides and finite difference time domain schemes have been used in physical modeling of spatially distributed systems. Both of them are known to provide exact modeling of ideal one-dimensional (1D) band-limited wave propagation, and both of them can be composed to approximate two-dimensional (2D) and three-dimensional (3D) mesh structures. Their equal capabilities in physical modeling have been shown for special cases and have been assumed to cover generalized cases as well. The ability to form mixed models by joining substructures of both classes through converter elements has been proposed recently. In this paper, we formulate a general digital signal processing (DSP)-oriented framework where the functional equivalence of these two approaches is systematically elaborated and the conditions of building mixed models are studied. An example of mixed modeling of a $2 \mathrm{D}$ waveguide is presented.
\end{abstract}

Keywords and phrases: acoustic signal processing, hybrid models, digital waveguides, scattering, FDTD model structures.

\section{INTRODUCTION}

Discrete-time simulation of spatially distributed acoustic systems for sound and voice synthesis finds its roots both in modeling of speech production and musical instruments. The Kelly-Lochbaum vocal tract model [1] introduced a onedimensional transmission line simulation of speech production with two-directional delay lines and scattering junctions for nonhomogeneous vocal tract profiles. Delay sections discretize the d'Alembert solution of the wave equation [2] and the scattering junctions implement the acoustic continuity laws of pressure and volume velocity in a tube of varying diameter. Further simplification led to the synthesis models used as the basis for linear prediction of speech [3].

A similar modeling approach to musical instruments, such as string and wind instruments, was formulated later and named the technique of digital waveguides (DWGs) $[4,5]$. For computational efficiency reasons, in DWGs twodirectional delay lines are often reduced to single delay loops [6]. DWGs have been further discussed in two-dimensional (2D) and three-dimensional (3D) modeling $[5,7,8,9,10]$, combined sometimes with a finite difference approach into DWG meshes.
Finite difference schemes [11] were introduced to the simulation of vibrating string as a numerical integration solution of the wave equation $[12,13]$, and the approach has been developed further for example in [14] as a finite difference time domain (FDTD) simulation. The second-order finite difference scheme including propagation losses was formulated as a digital filter structure in [15], and its stability issues were discussed in [16]. This particular structure is the main focus of the finite difference discussions in the rest of this paper and we will refer to it as the FDTD model structure.

DWG and FDTD approaches to discrete-time simulation of spatially distributed systems show a high degree of functional equivalence. As discussed in [5], in the onedimensional band-limited case, the ideal wave propagation can be exactly modeled by both methods. The basic difference is that the FDTD model structures process the signals as they are, whereas DWGs process their wave decomposition. There are other known differences between DWGs and FDTD model structures. One of them is the instabilities ("spurious" responses) found in FDTD model structures, but not in DWGs, to specific excitations. Another difference is the numeric behavior in finite precision computation. 
Comparison of these two different paradigms has been developed further in $[10,17,18]$. In [17], the interesting and important possibility of building mixed models with submodels of DWG and FDTD types was introduced and generalized to elements with arbitrary wave impedances in [18]. The problem of functional comparison and compatibility analysis has remained, however, and is the topic of this paper.

The rest of the paper is organized as follows. Section 2 provides the background information and notation that will be used in the following sections. A summary of wavebased modeling and finite difference modeling is also included in this section. Section 3 provides the derivation of the FDTD model structures, including the source terms, scattering, and the continuity laws. Based on the wave equation in the acoustical domain, this section highlights the functional equivalence of DWGs and FDTD model structures. It also presents a way of building mixed models. The formal proofs of equivalence are provided in "Appendix." Section 4 is devoted to real-time implementation of mixed models. Finally, Section 5 draws conclusions and indicates future directions.

\section{BACKGROUND}

Sound synthesis algorithms that simulate spatially distributed acoustic systems usually provide discrete-time solutions to a hyperbolic partial differential equation, that is, the wave equation. According to the domain of simulation, the variables correspond to different physical quantities. The physical variables may further be characterized by their mathematical nature. An across variable is defined here to describe a difference between two values of an irrotational potential function (a function that integrates or sums up to zero over closed trajectories), whereas a through variable is defined here to describe a solenoidal function (a quantity that integrates or sums-up to zero over closed surfaces). For example in the acoustical domain, the deviation from the steady-state pressure $p(\mathbf{x}, t)$ is an across variable and the volume velocity $u(\mathbf{x}, t)$ is a through variable, where $\mathbf{x}$ is the spatial vector variable and $t$ is the temporal scalar variable. Similarly, in the mechanical domain, the across variable is the force and the through variable is the velocity. The ratio of the through and across variables yields the impedance $Z$. The admittance is the inverse of $Z$, that is, $Y=1 / Z$.

In a one-dimensional (1D) medium, the spatial vector variable reduces to a scalar variable $x$, so that in a homogeneous, lossless, unbounded, and source-free medium the wave equation is written

$$
y_{t t}=c^{2} y_{x x}
$$

where $y$ is a physical variable, subscript $t t$ refers to the second partial derivative in time $t, x x$ to the second partial derivative in spatial variable $x$, and $c$ is speed of wavefront in the medium of interest. For example in the mechanical domain (e.g., vibrating string) we are primarily interested in transversal wave motion for which $c=\sqrt{T / \mu}$, where $T$ is tension force and $\mu$ is mass per unit length of the string [2]. The impedance is closely related to the tension $T$, mass density $\mu$, and the propagation speed $c$ and is given by $Z=\sqrt{T \mu}=T / c$. In the acoustical domain, the admittance is also related to the acoustical propagation speed $c$. For instance, the admittance of a tube with a constant cross-section area $A$ is given by

$$
Y=\frac{A}{\rho c},
$$

where $\rho$ is the gas density in the tube.

The two common forms of discretizing the wave equation for numerical simulation are through traveling wave solution and by finite difference formulation.

\subsection{Wave-based modeling}

The traveling wave formulation is based on the d'Alembert solution of propagation of two opposite direction waves, that is,

$$
y(x, t)=\vec{y}(x-c t)+\bar{y}(x+c t) .
$$

Here, the arrows denote the right-going and the left-going components of the total waveform. Assuming that the signals are bandlimited to half of sampling rate, we may sample the traveling waves without losing any information by selecting $T$ as the sample interval and $X$ the position interval between samples so that $T=X / c$. Sampling is applied in a discrete time-space grid in which $n$ and $k$ are related to time and position, respectively. The discretized version of (3) becomes [5]:

$$
y(k, n)=\vec{y}(k-n)+\bar{y}(k+n) .
$$

It follows that the wave propagation can be computed by updating state variables in two delay lines by

$$
\vec{y}_{k, n+1}=\vec{y}_{k-1, n}, \quad \bar{y}_{k, n+1}=\bar{y}_{k+1, n},
$$

that is, by simply shifting the samples to the right and left, respectively. The shift is implemented with a pair of delay lines, and this kind of discrete-time modeling is called DWG modeling [5]. Since the physical variables are split into directional wave components, we will refer to such models as $W$-models. According to (3) or (4), a single physical variable (either through or across) is computed by summing the traveling waves, whereas the other one may be computed implicitly via the impedance.

If the medium is nonhomogeneous, then the admittance varies as a function of the spatial variable. In this case, the energy transfer between the wave components should be computed according to Kirchhoff-type of continuity laws, ensuring that the total energy is preserved. These laws may be derived utilizing the irrotational and solenoidal nature of across and through variables, respectively. In the DWG equivalent, the change in $Y$ across a junction of the waveguide sections causes scattering and the scattering junctions of interconnected ports, with given admittances and wave variables, 
have to be formulated [5]. For instance, in a parallel junction of $N$ waveguides in the acoustical domain, the Kirchhoff constraints are

$$
\begin{gathered}
P_{1}=P_{2}=\cdots=P_{N}=P_{J}, \\
U_{1}+U_{2}+\cdots+U_{N}+U_{\text {ext }}=0
\end{gathered}
$$

where $P_{i}$ and $U_{i}$ are the total pressure and volume velocity of the $i$ th branch ${ }^{1}$, respectively, $P_{J}$ is the common pressure of coupled branches, and $U_{\text {ext }}$ is an external volume velocity to the junction. Such a junction is illustrated in Figure 1. When port pressures are represented by incoming wave components $P_{i}^{+}$, outgoing wave components by $P_{i}^{-}$, admittances attached to each port by $Y_{i}$, and

$$
P_{i}=P_{i}^{+}+P_{i}^{-}, \quad U_{i}^{+}=Y_{i} P_{i}^{+},
$$

the junction pressure $P_{J}$ can be obtained as

$$
P_{J}=\frac{1}{Y_{\mathrm{tot}}}\left(U_{\mathrm{ext}}+2 \sum_{i=1}^{N} Y_{i} P_{i}^{+}\right),
$$

where $Y_{\text {tot }}=\sum_{i=1}^{N} Y_{i}$ is the sum of all admittances to the junction. Outgoing pressure waves are obtained from (7) to yield $P_{i}^{-}=P_{J}-P_{i}^{+}$. The resulting junction, a $W$-node, is depicted in Figure 2. The delay lines or termination admittances (see appendix) are connected to the $W$-ports of a Wnode.

A useful addition to DWG theory is to adopt wave digital filters (WDF) $[10,19]$ as discrete-time simulators of lumped parameter elements. Being based on $\mathrm{W}$-modeling, they are computationally compatible with the W-type DWGs $[10,18$, 20].

\subsection{Finite difference modeling}

In the most commonly used way to discretize the wave equation by finite differences, the partial derivatives in (1) are approximated by centered differences. The centered difference approximation to the spatial partial derivative $y_{x}$ is given by [11]

$$
y_{x} \approx \frac{(y(x+\Delta x / 2, t)-y(x-\Delta x / 2, t))}{\Delta x},
$$

where $\Delta x$ is the spatial sampling interval. A similar expression is obtained for the temporal partial derivative, if $x$ is kept constant and $t$ is replaced by $t \pm \Delta t$, where $\Delta t$ is the discrete-time sampling interval. Iterating the difference approximations, second-order partial derivatives in (1) are approximated by

$$
\begin{aligned}
& y_{x x} \approx \frac{\left(y_{x+\Delta x, t}-2 y_{x, t}+y_{x-\Delta x, t}\right)}{\Delta x^{2}}, \\
& y_{t t} \approx \frac{\left(y_{x, t+\Delta t}-2 y_{x, t}+y_{x, t-\Delta t}\right)}{\Delta t^{2}},
\end{aligned}
$$

\footnotetext{
${ }^{1}$ Note that capital letters denote a transform variable. For instance, $P_{i}$ is the $z$-transform of the signal $p_{i}(n)$.
}

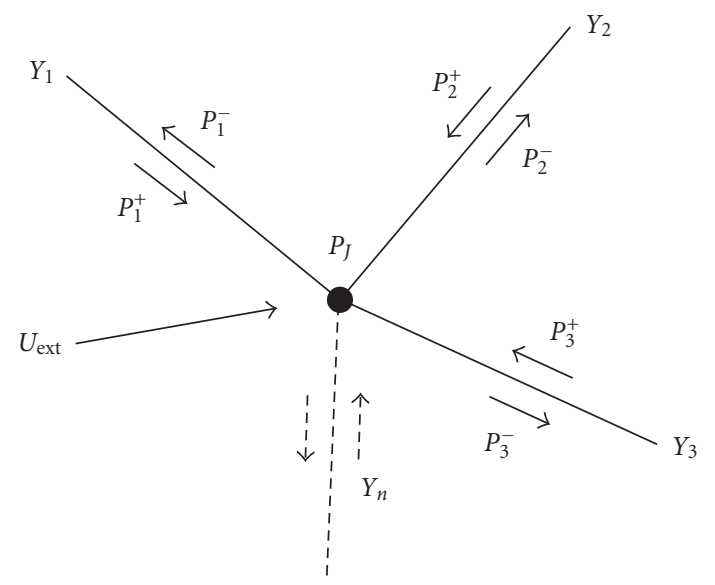

Figure 1: Parallel junction of admittances $Y_{i}$ with associated pressure waves indicated. A volume velocity input $U_{\text {ext }}$ is also attached.

where the short-hand notation $y_{x, t}$ is used instead of $y(x, t)$. By selecting $\Delta t=\Delta x / c$, and using index notation $k=x / \Delta x$ and $n=t / \Delta t,(10)$ result in

$$
y_{k, n+1}=y_{k-1, n}+y_{k+1, n}-y_{k, n-1}
$$

From (11) we can see that a new sample $y_{k, n+1}$ at position $k$ and time index $n+1$ is computed as the sum of its neighboring position values minus the value at the position itself one sample period earlier. Since $y_{k, n+1}$ is a physical variable, we will refer to models based on finite differences as $K$-models, with a reference to Kirchhoff type of physical variables.

\section{FORMULATION OF THE FDTD MODEL STRUCTURE}

The equivalence of the traveling wave and the finite difference solution of the ideal wave equation (given in (5) and (11), respectively) has been shown, for instance, in [5]. Based on this functional equivalence, (11) has been previously expanded without a formal derivation to a scattering junction with arbitrary port impedances, where (8) is used as a template for the expansion [18]. The resulting FDTD model structure is illustrated in Figure 3 for a three-port junction. A comparison of the FDTD model structure in Figure 3 and the DWG scattering junction in Figure 2 reveals the functional similarities of the two methods. However, a formal, generalized, and unified derivation of the FDTD model structure without an explicit reference to the DWG method remains to be presented. This section presents such a derivation based on the equations of motion of the gas in a tube. Note that, because of the analogy between different physical domains, once the formulation is derived, it can be used in different domains as well. Therefore, the derivation below is not limited to the acoustical domain and the resulting structure can also be used in other domains.

\subsection{Source terms}

In order to explain the excitation $U_{\text {ext }}$ and the associated filter $H(z)=1-z^{-2}$ in Figure 3, we consider a piece of tube of 

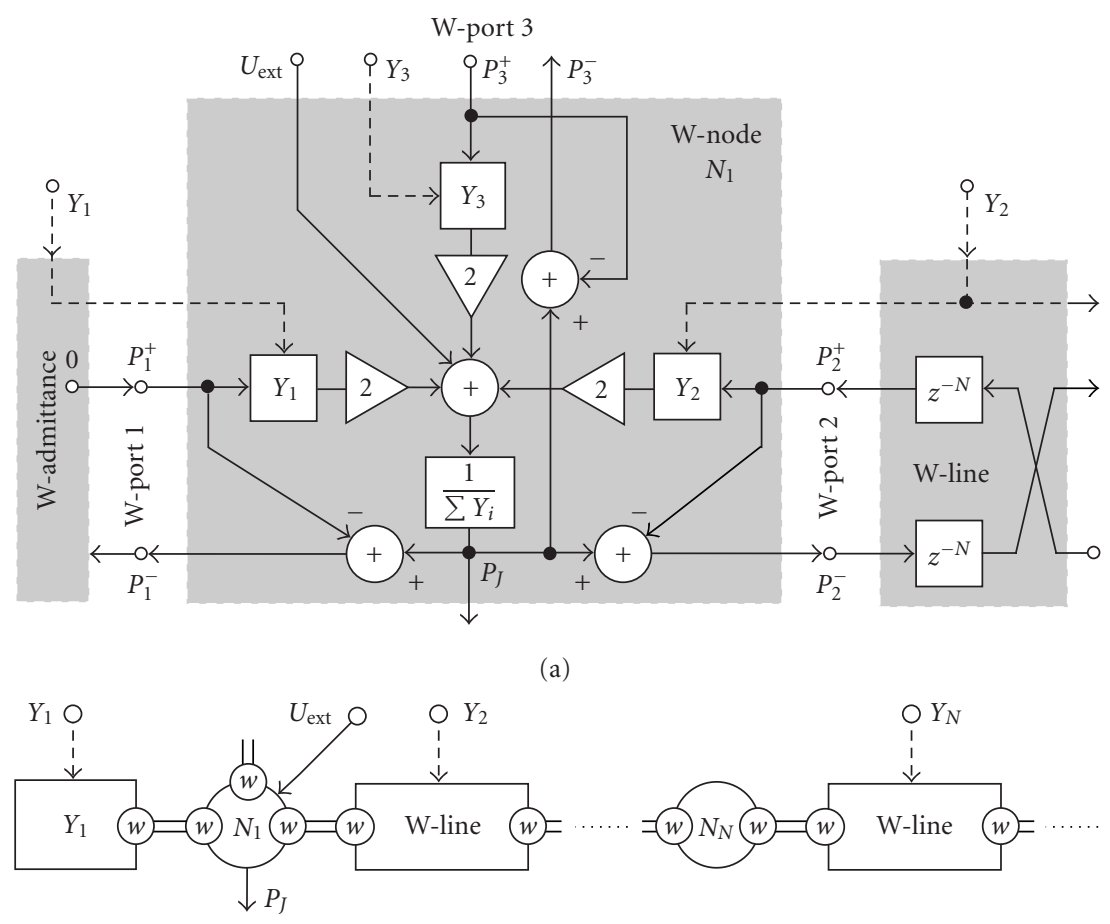

(b)

FiguRE 2: (a) $N$-port scattering junction (three ports are shown) of ports with admittances $Y_{i}$. Incoming and outgoing pressure waves are $P_{i}^{+}$and $P_{i}^{-}$, respectively. W-port 1 is terminated by admittance $Y_{1}$. (b) Abstract representation of the W-node in (a).

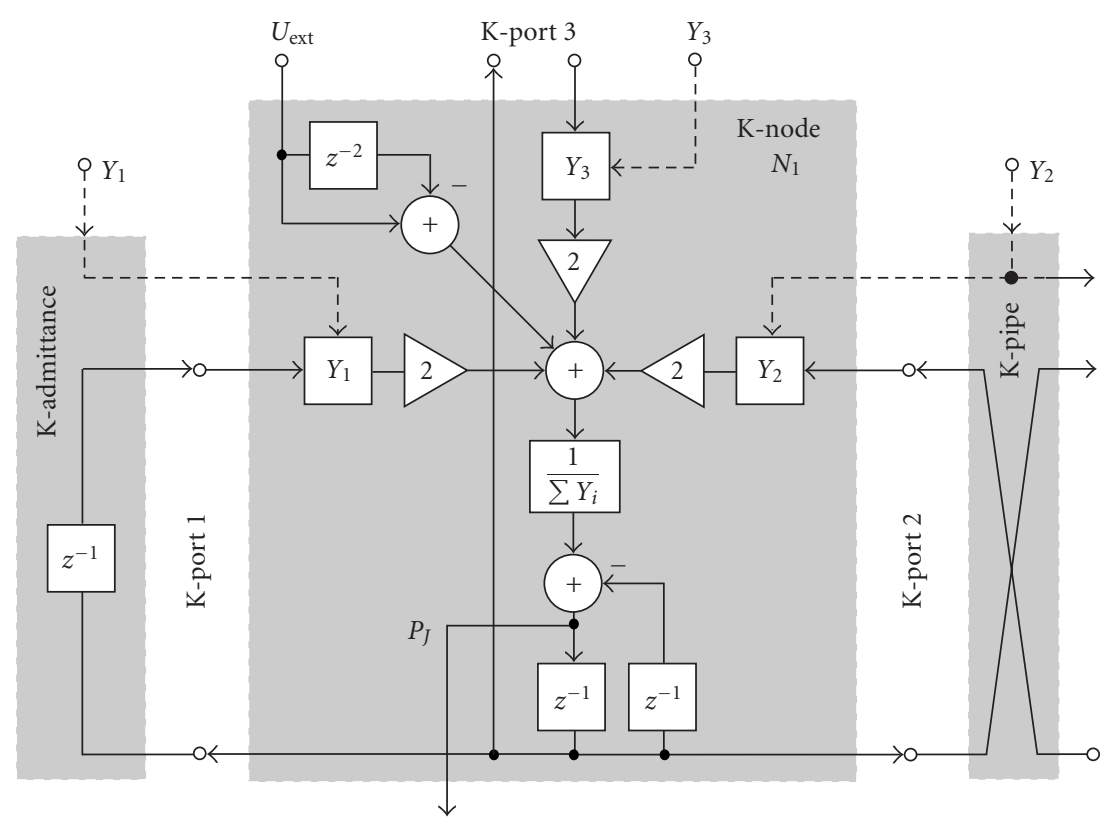

(a)

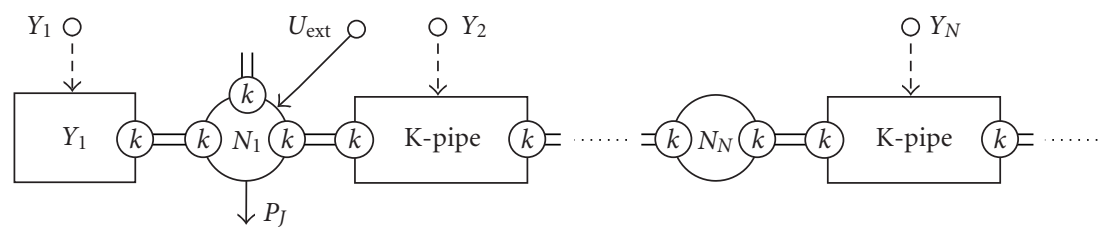

(b)

Figure 3: (a) Digital filter structure for finite difference approximation of a three-port scattering node with port admittances $Y_{i}$. Only total velocity $P_{J}$ (K-variable) is explicitly available. (b) Abstract representation of the K-node in (a). 
constant cross-sectional area $A$ that includes an ideal volume velocity source $s(t)$. The pressure $p$ and volume velocity $u$ (the variables in the acoustical domain, as explained in the previous section) satisfy the following PDE set:

$$
\rho \frac{\partial u}{\partial t}+A \frac{\partial p}{\partial x}=0 \quad \frac{A}{\rho c^{2}} \frac{\partial p}{\partial t}+\frac{\partial u}{\partial x}=s,
$$

where $\rho$ is the gas density and $c$ is the propagation speed. This set may be combined to yield a single PDE in $p$ and the source term

$$
\frac{\partial^{2} p}{\partial t^{2}}-\frac{\rho c^{2}}{A} \frac{\partial s}{\partial t}=c^{2} \frac{\partial^{2} p}{\partial x^{2}}
$$

Defining

$$
s(t)=\frac{1}{2}\left(s\left(t-\frac{\Delta t}{2}\right)+s\left(t+\frac{\Delta t}{2}\right)\right)+O\left(\Delta t^{2}\right),
$$

using index notation $k=x / \Delta x$ and $n=t / \Delta t$, and applying centered differences (see Section 2.2) to (13) with $\Delta x / \Delta t=c$ yields the following difference equation

$$
\begin{aligned}
p_{k}(n+1)= & p_{k+1}(n)+p_{k-1}(n)-p_{k}(n-1) \\
& +\frac{\rho c \Delta x}{2 A}\left(s_{k}(n+1)-s_{k}(n-1)\right) .
\end{aligned}
$$

Note that $\rho c / A$ is the acoustic impedance that converts the volume velocity source $s(t)$ to the pressure. Since the model output is the pressure at the time step $n+1$, it follows that the source is delayed two samples, subtracted from its current value, and scaled, corresponding to the filter $1-z^{-2}$ for $U_{\text {ext }}$ in Figure 3.

\subsection{Admittance discontinuity and scattering}

Now consider an unbounded, source-free tube with a crosssection $A(x)$ that is a smooth real function of spatial variable $x$. In this case, the governing PDEs can be combined into a single PDE in the pressure alone [10],

$$
\frac{\partial^{2} p}{\partial t^{2}}=\frac{c^{2}}{A(x)} \frac{\partial}{\partial x}\left(A(x) \frac{\partial p}{\partial x}\right)
$$

which is the Webster horn equation. Discretizing this equation by centered differences yields the following difference equation

$$
\begin{aligned}
& \frac{p_{k}(n+1)-2 p_{k}(n)+p_{k}(n-1)}{\Delta t^{2}} \\
& =\frac{c^{2}}{A_{k}} \frac{A_{k+1 / 2}\left(p_{k+1}(n)-p_{k}(n)\right)-A_{k-1 / 2}\left(p_{k}(n)-p_{k-1}(n)\right)}{\Delta x^{2}},
\end{aligned}
$$

where $A_{k}=A(k \Delta x)$. By selecting $\Delta x=c \Delta t$ and using the approximation

$$
A_{k}=\frac{1}{2}\left(A_{k-1 / 2}+A_{k+1 / 2}\right)+O\left(\Delta x^{2}\right)
$$

twice, (17) becomes

$$
\begin{aligned}
p_{k}(n+1)+p_{k}(n-1) & \\
\quad= & \frac{2}{A_{k-1 / 2}+A_{k+1 / 2}}\left(A_{k-1 / 2} p_{k-1}(n)+A_{k+1 / 2} p_{k+1}(n)\right) .
\end{aligned}
$$

Finally, by defining $Y_{k-1}=A_{k-1 / 2} / \rho c$ we obtain

$$
\begin{aligned}
p_{k}(n+1)+p_{k}(n-1) & \\
& =\frac{2}{Y_{\text {tot }}}\left(Y_{k-1} p_{k-1}(n)+Y_{k+1} p_{k+1}(n)\right),
\end{aligned}
$$

where the term $Y_{\text {tot }}=Y_{k-1}+Y_{k+1}$ may be interpreted as the sum of all admittances connected to the $k$ th cell. This recursion is implemented with the filter structure illustrated in Figure 4. The output of the structure is the junction pressure $p_{J, k}(n)$. It is worth to note that $(20)$ is functionally the same as the DWG scattering representation given in (8), if the admittances are real. A more general case of complex admittances has been considered in the appendix. Whereas the DWG formulation can easily be extended to $N$-port junctions, this extension is not necessarily possible for a $\mathrm{K}$-model, where the continuity laws are generally not satisfied. In the next subsection, we investigate the continuity laws within the FDTD model structure.

\subsection{Continuity laws}

We denote the pressure across the impedance $1 / \sum Y_{i}$ as $p_{\mathrm{a}}(n)$, and the volume velocity through the same impedance as $u_{\mathrm{t}}(n)$, with a reference to Figure 4 . According to these notations, Ohm's law in the acoustical domain yields

$$
p_{\mathrm{a}}(n)=\frac{u_{\mathrm{t}}(n)}{Y_{\mathrm{tot}}},
$$

whereas the Kirchhoff continuity laws can be written as

$$
\begin{gathered}
p_{\mathrm{a}}(n)=p_{k}(n+1)+p_{k}(n-1), \\
u_{\mathrm{t}}(n)=2 Y_{k-1} p_{k-1}(n)+2 Y_{k+1} p_{k+1}(n) .
\end{gathered}
$$

Inserting (21) into (23) eliminates $u_{t}(n)$, and the result may be combined with (22) to give the following equation for combined continuity laws:

$$
\begin{aligned}
p_{k}(n & +1)+p_{k}(n-1) \\
& =\frac{2}{Y_{\text {tot }}}\left(Y_{k-1} p_{k-1}(n)+Y_{k+1} p_{k+1}(n)\right) .
\end{aligned}
$$

This relation is exactly the recursion of the FDTD model structure given in (20), but obtained here solely from the continuity laws. We thus conclude that the continuity laws are automatically satisfied by the FDTD model structure of Figure 4.

It is worth to note that more ports may be added to the structure without violating the continuity laws for any number of linear, time-invariant (LTI) admittances, as long as $Y_{\text {tot }}=\sum Y_{i}$. For $N$ ports connected to the $i$ th cell, (23) becomes

$$
U_{\mathrm{t}}=2 \sum_{i=1}^{N} z^{-1} Y_{i} P_{J, i}
$$




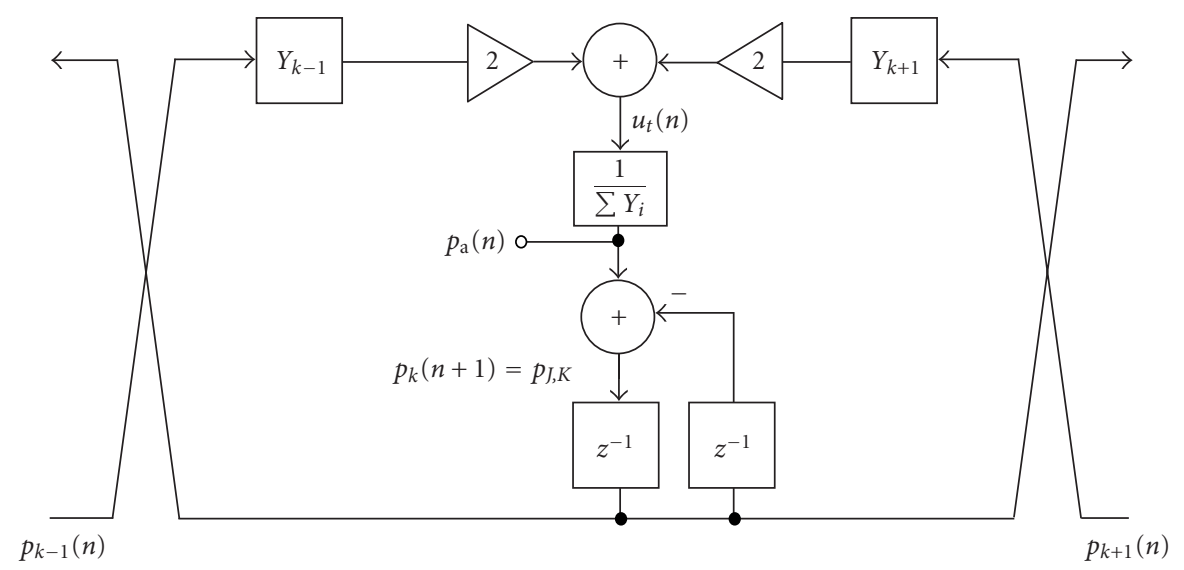

FIGURE 4: Digital filter structure for finite difference approximation of an unbounded, source-free tube with a spatially varying cross section.

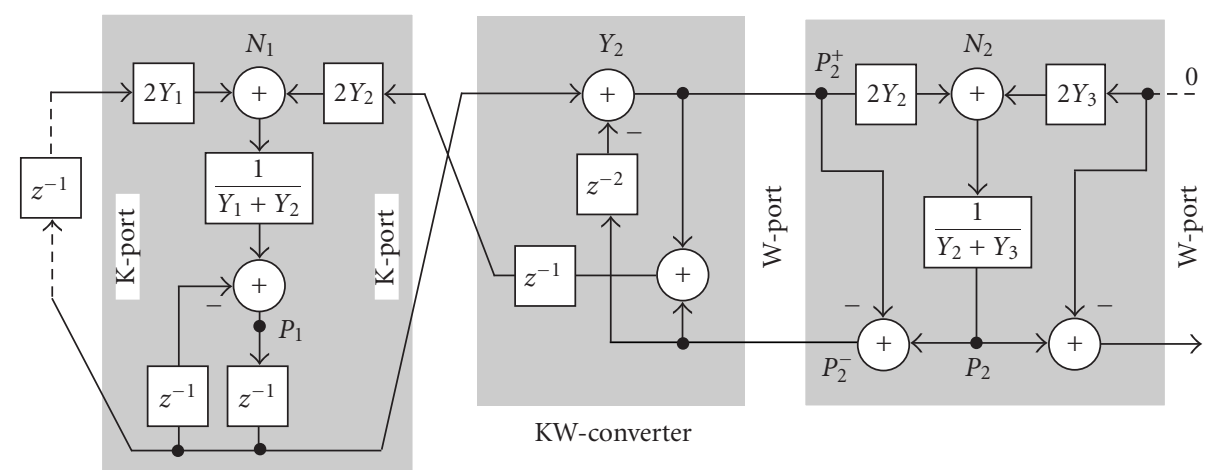

FIGURE 5: FDTD node (left) and a DWG node (right) forming a part of a hybrid waveguide. There is a KW-converter between K- and Wmodels. $Y_{i}$ are wave admittances of W-lines, K-pipes, and KW-converter between junction nodes. $P_{1}$ and $P_{2}$ are the junction pressures of the $\mathrm{K}$-node and $\mathrm{W}$-node, respectively.

and the recursion in (24) can be expressed in $z$-domain as

$$
P_{J, k}+z^{-2} P_{J, k}=\frac{2}{\sum Y_{i}} \sum_{i=1}^{N} z^{-1} Y_{i} P_{J, i}
$$

The superposition of the excitation block in (14) and the $\mathrm{N}$-port formulation above completes the formulation of the FDTD model structure. In particular, by setting $N=3$ the digital filter structure in Figure 3 is obtained.

\subsection{Construction of mixed models}

An essential difference between DWGs of Figure 2 and FDTD model structures of Figure 3 is that while DWG junctions are connected through two-directional delay lines ( $W$-lines), FDTD nodes have two unit delays of internal memory and delay-free K-pipes connecting ports between nodes. These junction nodes and ports are thus not directly compatible. The next question is the possibility to interface these submodels. The interconnection of a lossy FDTD model structure and a similar DWG has been tackled in [17]. A proper interconnection element (converter) has been proposed for the resulting hybrid model in this special case. A generalization has been proposed in [18], which allows to make any hybrid model of K-elements (FDTD) and W-elements having arbitrary wave admittances/impedances at their ports (see also [21]).

Here, we derive how a hybrid model (shown in Figure 5) can be constructed in a 1D waveguide between a K-node $N_{1}$ (left) and a W-node $N_{2}$ (right), aligned with the spatial grids $k=1$ and 2 , respectively. The derivation is based on the fact that the junction pressures are available in both types of nodes, but in the DWG case not at the W-ports.

If $N_{1}$ and $N_{2}$ would be both W-nodes (see Figure 8 in the appendix), the traveling wave entering into the node $N_{2}$ could be calculated as

$$
P_{2}^{+}=z^{-1} P_{1}^{-}=z^{-1}\left(P_{1}-z^{-1} P_{2}^{-}\right)=z^{-1} P_{1}-z^{-2} P_{2}^{-} .
$$

Note that $P_{1}$ is available in the K-node $N_{1}$ in Figure 5. Conversely, if $N_{1}$ and $N_{2}$ would be both K-nodes, the junction pressure $z^{-1} P_{2}$ would be needed for calculation of $P_{1}$ (see Figure 10 in the appendix). Although $P_{2}$ is implicitly available in $N_{2}$, it can also be obtained by summing up the wave 


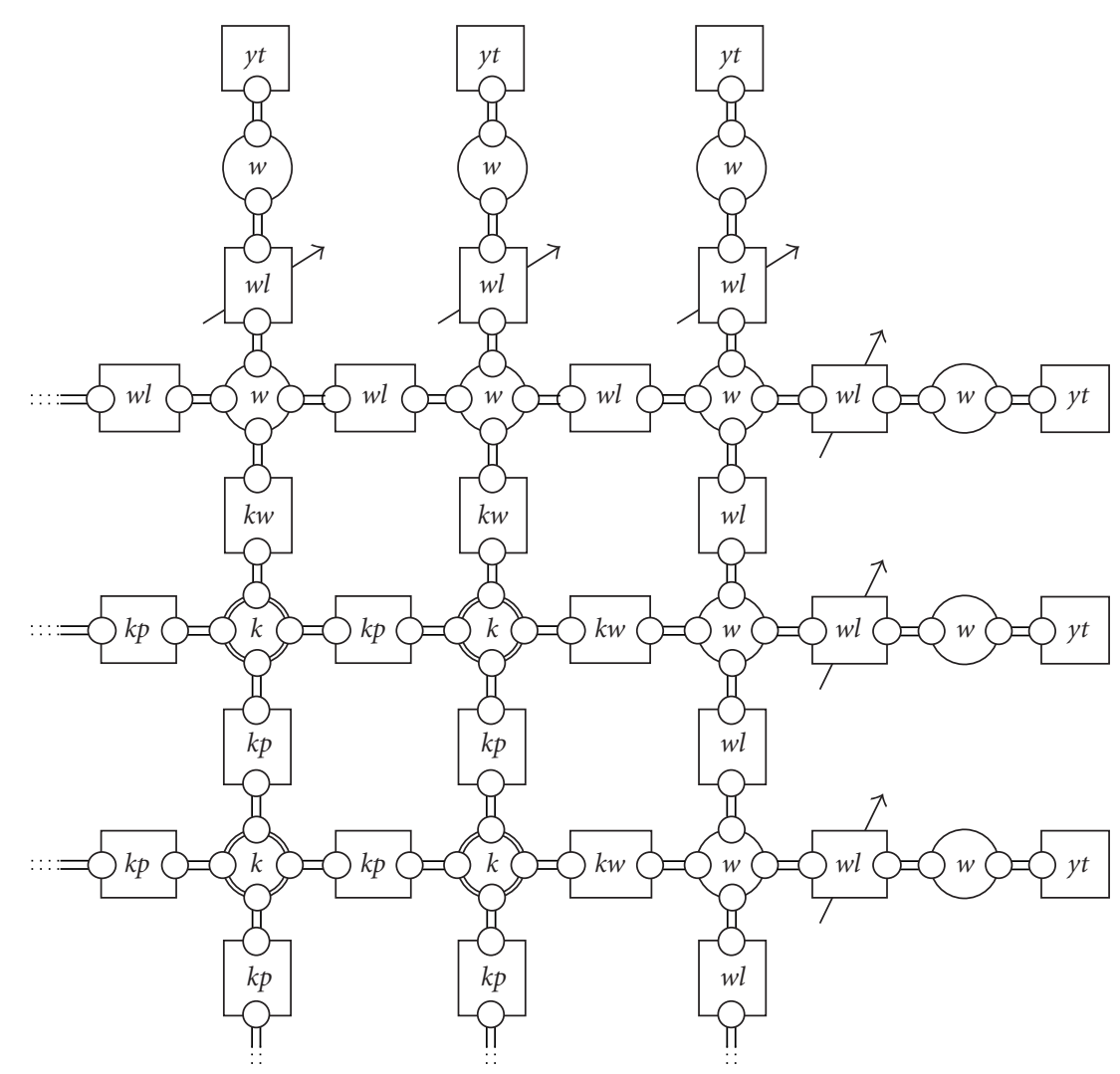

Figure 6: Part of a 2D waveguide mesh composed of (a) K-type FDTD elements (left bottom): K-pipes ( $k p$ ) and K-nodes ( $k$ ), (b) W-type DWG elements (top and right): delay-controllable W-lines $(w l)$, W-nodes $(w)$, and terminating admittances $(y t)$, and $(\mathrm{c})$ converter elements $(k w)$ to connect $\mathrm{K}$ - and $\mathrm{W}$-type elements into a mixed model.

components within the converter

$$
z^{-1} P_{2}=z^{-1}\left(P_{2}^{+}+P_{2}^{-}\right) .
$$

Equation (27) may be inserted in (28) to yield the following transfer matrix of the 2-port KW-converter element

$$
\left[\begin{array}{c}
P_{2}^{+} \\
z^{-1} P_{2}
\end{array}\right]=\left[\begin{array}{cc}
1 & -z^{-2} \\
1 & \left(1-z^{-2}\right)
\end{array}\right]\left[\begin{array}{c}
z^{-1} P_{1} \\
P_{2}^{-}
\end{array}\right] .
$$

The KW-converter in Figure 5 essentially performs the calculations given in (29) and interconnects the K-type port of an FDTD node and the W-type port of a DWG node. The signal behavior in a mixed modeling structure is further investigated in the appendix.

\section{IMPLEMENTATION OF MIXED MODELS}

The functional equivalence and mixed modeling paradigm of DWGs and FDTDs presented above allows for flexible building of physical models from K- and W-type of substructures. In this way, it is possible to exploit the advantages of each type. In this section, we will explore a simple example of digital waveguide model that shows how the mixed models can be built. Before that, a short discussion on the pros and cons of the different paradigms in practical realizations is presented.

\subsection{K-modeling versus $\boldsymbol{W}$-modeling, pros and cons}

An advantage of $\mathrm{W}$-modeling is in its numerical robustness. By proper formulation, the stability is guaranteed also with fixed-point arithmetics [5, 19]. Another useful property is the relatively straightforward way of using fractional delays [22] when building digital waveguides, which makes for example tuning and run time variation of musical instrument models convenient. In general, it seems that $\mathrm{W}$-modeling is the right choice in most $1 \mathrm{D}$ cases.

The advantages of K-modeling by FDTD waveguides are found when realizing mesh-like structures, such as $2 \mathrm{D}$ and $3 \mathrm{D}$ meshes $[7,8]$. In such cases, the number of unit delays (memory positions) is two for any dimensionality, while for a DWG mesh it is two times the dimensionality of the mesh. A disadvantage of FDTDs is their inherent lack of numerical robustness and tendency of instability for signal frequencies near DC and the Nyquist frequency. Furthermore, FDTD junction nodes cannot be made memoryless, which may be a limitation in nonlinear and parametrically varying models.

\subsection{D waveguide mesh case}

Figure 6 illustrates a part of a 2D mixed model structure that is based on a rectangular FDTD waveguide mesh for efficient and memory-saving computation and DWG elements at boundaries. Such model could be for example a membrane 
of a drum or in a $3 \mathrm{D}$ case a room enclosed by walls. When there is need to attach W-type termination admittances to the model or to vary the propagation delays within the system, a change from K-elements to W-elements through converters is a useful property. Furthermore, variable-length delays can be used, for example, for passive nonlinearities at the terminations to simulate gongs and other instruments where nonlinear mode coupling takes place [23]. The same principle can be used to simulate shock waves in brass instrument bores [24]. In such cases, the delay lengths are made dependent on the signal value passing through the delay elements.

In Figure 6, the elements denoted by $k p$ are K-type pipes between $\mathrm{K}$-type nodes. Elements $k w$ are $\mathrm{K}$-to-W converters and elements $w l$ are $\mathrm{W}$-lines, where the arrows indicate that they are controllable fractional delays. Elements $y t$ are terminating admittances. In a general case, scattering can be controlled by varying the admittances, although the computational efficiency is improved if the admittances are made equal. In a modern PC, a 2D mesh of a few hundred elements can run in real time at full audio rate. By decimated computation, bigger models can be computed if a lower cutoff frequency is permitted, allowing large physical dimensions of the mesh.

\subsection{Mixed modeling in BlockCompiler}

The development of the K- and W-models above has led to a systematic formulation of computational elements for both paradigms and mixed modeling. The $\mathrm{W}$-lines and $\mathrm{K}$-pipes as well as related junction nodes are useful abstractions for a formal specification of model implementation. We have developed a software tool for physical modeling called the BlockCompiler [20] that is designed in particular for flexible modeling and efficient real-time computation of the models.

The BlockCompiler contains two levels: (a) model creation and (b) model implementation. The model creation level is written in the Common Lisp programming language for maximal flexibility in symbolic object-based manipulation of model structures. A set of DSP-oriented and physics-oriented computational blocks are available. New block classes can be created either as macro classes composed of predefined elementary blocks or by writing new elementary blocks. The blocks are connected through ports: inputs and outputs for DSP blocks and K- or W-type ports for physical blocks. A full interconnected model is called a patch.

The model implementation level is a code generator that does the scheduling of the blocks, writes $\mathrm{C}$ source code into a file, compiles it on the fly, and allows for streaming sound in real time or computation by stepping in a sample-bysample mode. The $\mathrm{C}$ code can also be exported to other platforms, such as the Mustajuuri audio platform [25] and pd [26]. Sound examples of mixed models can be found at http://www.acoustics.hut.fi/demos/waveguide-modeling/.

\section{SUMMARY AND CONCLUSIONS}

This paper has presented a formulation of a specific FDTD model structure and showed its functional equivalence to the
DWGs. Furthermore, an example of mixed models consisting of FDTD and DWG blocks and converter elements is reported. The formulation allows for high flexibility in building $1 \mathrm{D}$ or higher dimensional physical models from interconnected blocks.

The DWG method is used as a primary example to the wave-based methods in this paper. Naturally, the KWconverter formulation is applicable to any $\mathrm{W}$-method, such as the wave digital filters (WDFs) [19]. In the future, we plan to extend our examples to include WDF excitation blocks. Other important future directions are the analysis of the dynamic behavior of parametrically varying hybrid models, as well as benchmark tests for computational costs of the proposed structures.

Matlab scripts and demos related to DWGs and FDTDs can be found at http://www.acoustics.hut.fi/demos/ waveguide-modeling/.

\section{APPENDIX}

\section{A. PROOFS OF EQUIVALENCE}

The proofs of functional equivalence between the DWG and FDTD formulations used in this article are given below. The approach useful for this can be based on the Thevenin and Norton theorems [27].

\section{A.1. Termination in a DWG network}

Passive termination of a DWG junction port by a given admittance $Y$ is equivalent to attaching a delay line of infinite length and wave admittance $Y$. In the DWG case, this means an infinite long sequence of admittance-matched unit delay lines. Since there is no back-scattering in finite time, we can use the left-side port termination of Figure 2, with zero volume velocity in input terminal. Thus, admittance filter $Y_{1}$ is not needed in computation, it has only to be included in making the filter $1 / \sum Y_{i}$.

\section{A.2. Termination in an FDTD network}

Deriving the passive port termination for an FDTD junction is not as obvious as for a DWG junction. We can apply again an infinitely long sequence of admittance-matched FDTD sections, as depicted in Figure 7 on the left-hand side. With the notations given and $z$-transforms of variables and admittances we can denote

$$
\begin{gathered}
P_{0}=\frac{2 Y_{1}}{\sum Y_{i}} P_{-1} z^{-1}+\frac{2}{\sum Y_{i}} \sum_{i=2}^{M} Y_{i} P_{i} z^{-1}-P_{0} z^{-2}, \\
P_{-1}=P_{0} z^{-1}+P_{-2} z^{-1}-P_{-1} z^{-2}, \\
P_{-k}=P_{-k+1} z^{-1}+P_{-k-1} z^{-1}-P_{-k} z^{-2}, \quad \text { for } k<-1,
\end{gathered}
$$

where $P_{i}, i=1, \ldots, M$, are pressures of all $M$ neighboring junctions linked through admittances $Y_{i}$ to junction $i=0$, and $P_{k}$, where $k=0,-1,-2, \ldots$ are pressures in junctions between admittance-matched elements chained as termination of junction 0 . By applying (A.1c) to (A.1b) iteratively for 


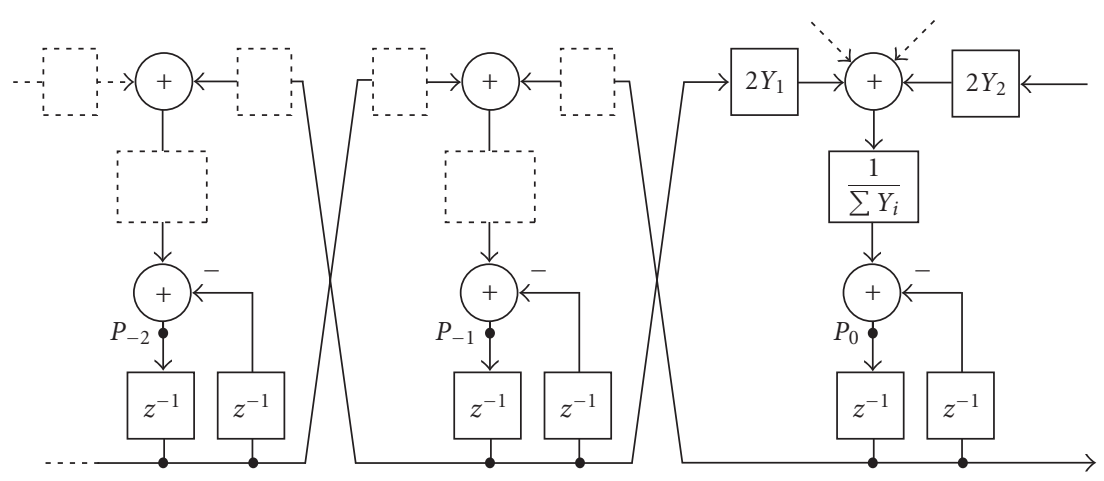

FIGURE 7: FDTD structure terminated by admittance-matched chain of FDTD elements on the left-hand side.

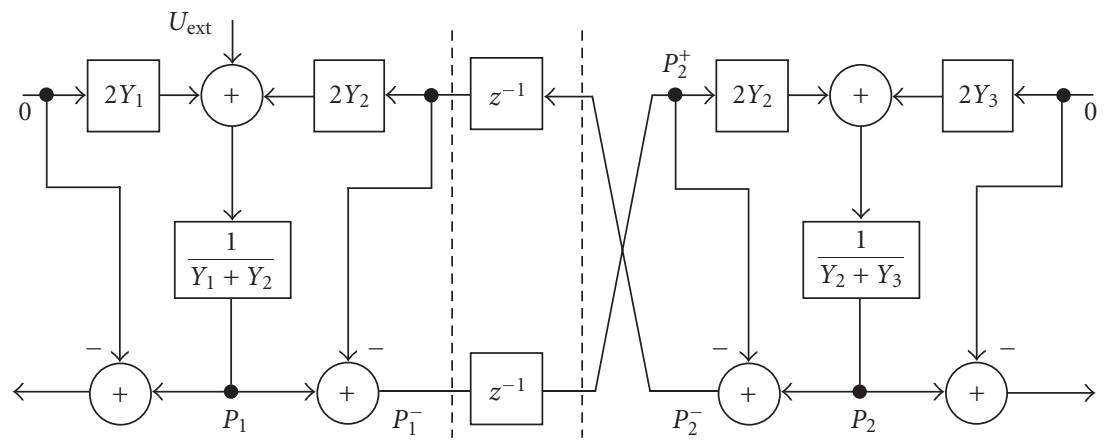

FIGURE 8: Structure for derivation of signal behavior in a DWG network.

$k=2, \ldots, N$ we get

$$
P_{-1}=P_{0} z^{-1}+P_{-N-1} z^{-N}-P_{-N} z^{-N-1} .
$$

When $N \rightarrow \infty$, the last two terms cease to have effect on $P_{-1}$ in any finite time span and they can thus be discarded. When the result $P_{-1}=P_{0} z^{-1}$ is used in (A.1a), we get

$$
P_{0}=\left\{\frac{2 Y_{1}}{\sum Y_{i}} P_{0} z^{-1}\right\} z^{-1}+\frac{2}{\sum Y_{i}} \sum_{i=2}^{M} Y_{i} P_{i} z^{-1}-P_{0} z^{-2},
$$

where the first term on the right-hand side can be interpreted as a way to implement the termination as a feedback through a unit delay as illustrated in Figure 3 for the left-hand port of the FDTD junction.

\section{A.3. Signal behavior in a DWG network}

Figure 8 illustrates a case where an arbitrarily large interconnected DWG network is reduced so that only two scattering junctions, connected through unit delay line of wave admittance $Y_{2}$, are shown explicitly. Norton equivalent source $U_{\text {ext }}$ is feeding junction node 1 and an equivalent termination admittance is $Y_{1}$. Junction node 2 is terminated by a Norton equivalent admittance $Y_{3}$. Now, we derive the signal propagation from $U_{\text {ext }}$ to junction pressure $P_{1}$ and transmission ratio between pressures $P_{2}$ and $P_{1}$. If these "transfer functions" are equal for the DWG, the FDTD, and the mixed case with KW-converter, the models are functionally equivalent

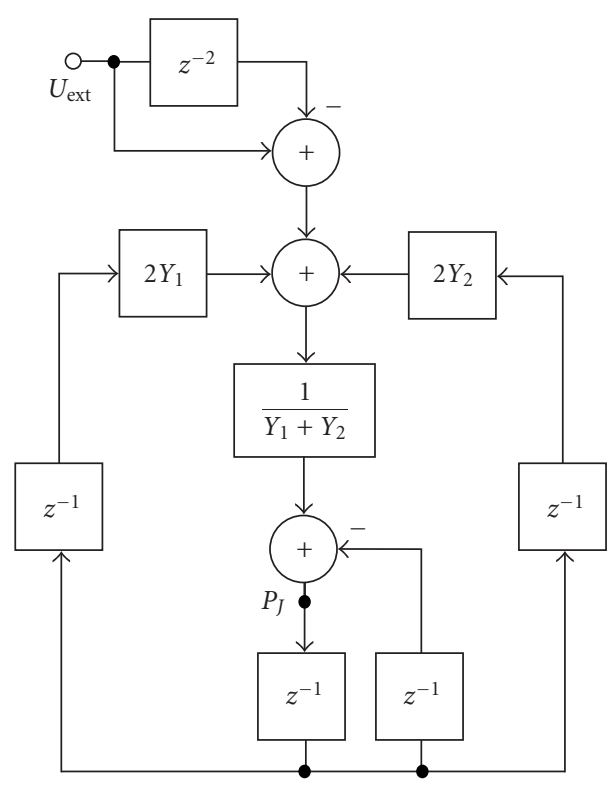

FIGURE 9: FDTD structure for derivation of volume velocity source $\left(U_{\text {ext }}\right)$ to junction pressure $\left(P_{J}\right)$ transfer function.

for any topologies and parametric values equivalent between these cases. This is due to the superposition principle and the Norton theorem. 


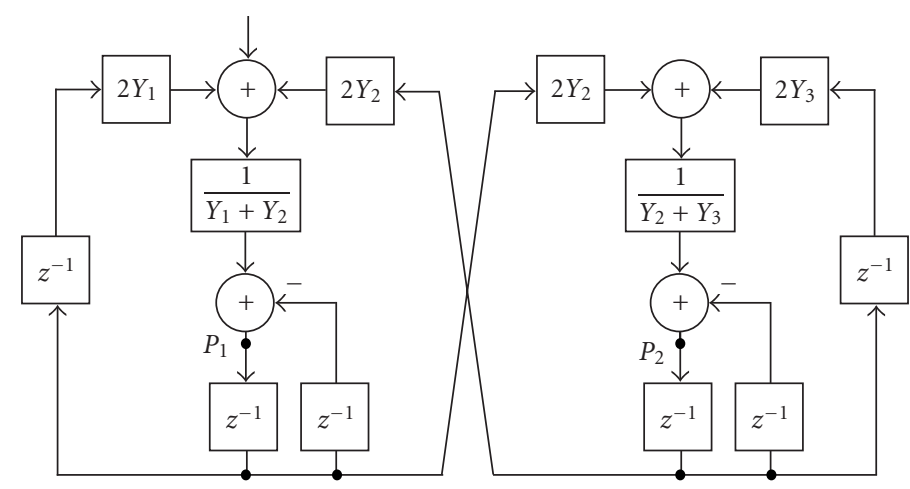

FigURE 10: FDTD structure for derivation of signal relation between two junction pressures.

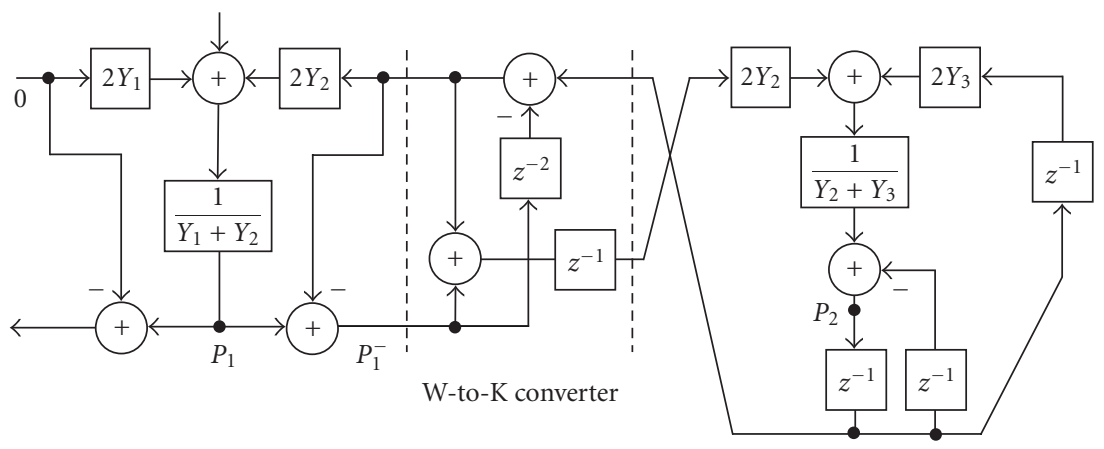

FIGURE 11: Mixed modeling structure for derivation of DWG to FDTD pressure relation.

From Figure 8, we can write directly for the propagation of equivalent source $U_{\text {ext }}$ to junction pressure $P_{1}$ as

$$
P_{1}=\frac{U_{\text {ext }}}{Y_{1}+Y_{2}} \text {. }
$$

Signal transmission ratio between $P_{2}$ and $P_{1}$ can be derived from the following set of equations (A.5a), (A.5b), and (A.5c):

$$
\begin{gathered}
P_{2}=\frac{2 Y_{2}}{Y_{2}+Y_{3}} P_{1}^{-} z^{-1} \\
P_{1}^{-}=P_{1}-P_{2}^{-} z^{-1} \\
P_{2}^{-}=P_{2}-P_{1}^{-} z^{-1}
\end{gathered}
$$

By eliminating wave variables $P_{1}^{-}$and $P_{2}^{-}$,

$$
\begin{gathered}
P_{1}^{-}=\frac{\left(P_{1}-P_{2} z^{-1}\right)}{\left(1-z^{-2}\right)}, \\
P_{2}^{-}=\frac{\left(P_{2}-P_{1} z^{-1}\right)}{\left(1-z^{-2}\right)}, \\
P_{2}=\frac{2 Y_{2}}{Y_{2}+Y_{3}}\left(P_{1}-P_{2} z^{-1}\right) \frac{z^{-1}}{1-z^{-2}}
\end{gathered}
$$

and by solving for $P_{2} / P_{1}$, we get

$$
\frac{P_{2}}{P_{1}}=\frac{2 Y_{2} z^{-1}}{Y_{2}+Y_{3}+\left(Y_{2}-Y_{3}\right) z^{-2}} \text {. }
$$

In the special case of admittance match $Y_{2}=Y_{3}$, we get $P_{2} / P_{1}$ $=z^{-1}$. Forms (A.4) and (A.7) are now the reference to prove equivalence with FDTD and mixed modeling cases.

\section{A.4. Signal behavior in an FDTD network}

Using notations in Figure 9, which shows a Norton's equivalent for an FDTD network, we can write

$$
\begin{aligned}
P_{J}= & \frac{U_{\text {ext }}}{Y_{1}+Y_{2}}\left(1-z^{-2}\right)-P_{J} z^{-2} \\
& +\frac{2 Y_{1}}{Y_{1}+Y_{2}} P_{J} z^{-2}+\frac{2 Y_{2}}{Y_{1}+Y_{2}} P_{J} z^{-2}
\end{aligned}
$$

that after simplification yields

$$
P_{J}=\frac{U_{\text {ext }}}{Y_{1}+Y_{2}},
$$

which is equivalent to the DWG form (A.4). Notice that form $\left(1-z^{-2}\right)$ in feeding $U_{\text {ext }}$ to the node has zeros on the unit circle for angles $n \pi$ ( $n$ is integer), compensating poles inherent in the FDTD backbone structure. This degrades numerical robustness of the structure around these frequencies.

For the structure of two FDTD nodes in Figure 10, we can write equation

$$
P_{2}=-P_{2} z^{-2}+\frac{2 Y_{3}}{Y_{2}+Y_{3}} P_{2} z^{-2}+\frac{2 Y_{2}}{Y_{2}+Y_{3}} P_{1} z^{-1},
$$


which simplifies to

$$
\frac{P_{2}}{P_{1}}=\frac{2 Y_{2} z^{-1}}{Y_{2}+Y_{3}+\left(Y_{2}-Y_{3}\right) z^{-2}}
$$

being equivalent to the DWG form (A.7). This completes proving the equivalence of the DWG and FDTD structures.

\section{A.5. Signal behavior in a mixed modeling structure}

To prove the equivalence of signal behavior also in the mixed modeling structure of Figure 5 with a KW-adaptor, we have to analyze the junction signal relations in both directions. We first prove the equivalence in the FDTD to DWG direction. According to Figure 5, we can write

$$
\begin{gathered}
P_{2}=\frac{2 Y_{2}}{Y_{2}+Y_{3}} P_{1} z^{-1}-\frac{2 Y_{2}}{Y_{2}+Y_{3}} P_{2}^{-} z^{-2} \\
P_{2}^{-}=P_{2}-\left(P_{1} z^{-1}-P_{2}^{-} z^{-2}\right)
\end{gathered}
$$

Eliminating $P_{2}^{-}$and solving for $P_{2} / P_{1}$ yields again form (A.7), proving the equivalence.

According to Figure 11, we can analyze signal relationship in the DWG to FDTD direction by writing

$$
\begin{aligned}
P_{2}= & \frac{2 Y_{3}}{Y_{2}+Y_{3}} P_{2} z^{-2}-P_{2} z^{-2} \\
& -\frac{2 Y_{2}}{Y_{2}+Y_{3}}\left(P_{1}^{-}-P_{1}^{-} z^{-2}+P_{2} z^{-1}\right) z^{-1}, \\
P_{1}^{-}= & P_{1}-\left(P_{2} z^{-1}-P_{1}^{-} z^{-2}\right) .
\end{aligned}
$$

By eliminating $P_{1}^{-}$and solving for $P_{2} / P_{1}$, we get again form (A.7). This concludes proving the equivalence of the mixed modeling case to corresponding DWG and thus also to FDTD structures.

\section{ACKNOWLEDGMENTS}

This work is part of the Algorithms for the Modelling of Acoustic Interactions (ALMA) project (IST-2001-33059) and has been supported by the Academy of Finland as a part of the project "Technology for Audio and Speech Processing" (SA 53537).

\section{REFERENCES}

[1] J. L. Kelly and C. C. Lochbaum, "Speech synthesis," in Proc. 4th International Congress on Acoustics, pp. 1-4, Copenhagen, Denmark, September 1962.

[2] N. H. Fletcher and T. D. Rossing, The Physics of Musical Instruments, Springer-Verlag, New York, NY, USA, 2nd edition, 1998.

[3] J. D. Markel and A. H. Gray, Linear Prediction of Speech, Springer-Verlag, New York, NY, USA, 1976.

[4] J. O. Smith, "Physical modeling using digital waveguides," Computer Music Journal, vol. 16, no. 4, pp. 74-91, 1992.

[5] J. O. Smith, "Principles of digital waveguide models of musical instruments," in Applications of Digital Signal Processing to Audio and Acoustics, M. Kahrs and K. Brandenburg, Eds., pp. 417-466, Kluwer Academic Publishers, Boston, Mass, USA, 1998.
[6] M. Karjalainen, V. Välimäki, and T. Tolonen, "Plucked-string models: From the Karplus-Strong algorithm to digital waveguides and beyond," Computer Music Journal, vol. 22, no. 3, pp. 17-32, 1998.

[7] S. A. Van Duyne and J. O. Smith, "Physical modeling with the 2-D digital waveguide mesh," in Proc. International Computer Music Conference, pp. 40-47, Tokyo, Japan, September 1993.

[8] L. Savioja, T. J. Rinne, and T. Takala, "Simulation of room acoustics with a 3-D finite difference mesh," in Proc. International Computer Music Conference, pp. 463-466, Aarhus, Denmark, September 1994.

[9] L. Savioja, Modeling techniques for virtual acoustics, Ph.D. thesis, Helsinki University of Technology, Espoo, Finland, 1999.

[10] S. D. Bilbao, Wave and scattering methods for the numerical integration of partial differential equations, Ph.D. thesis, Stanford University, Stanford, Calif, USA, May 2001.

[11] J. C. Strikwerda, Finite Difference Schemes and Partial Differential Equations, Wadsworth and Brooks/Cole, Pacific Grove, Calif, USA, 1989.

[12] L. Hiller and P. Ruiz, "Synthesizing musical sounds by solving the wave equation for vibrating objects: Part 1," Journal of the Audio Engineering Society, vol. 19, no. 6, pp. 462-470, 1971.

[13] L. Hiller and P. Ruiz, "Synthesizing musical sounds by solving the wave equation for vibrating objects: Part 2," Journal of the Audio Engineering Society, vol. 19, no. 7, pp. 542-551, 1971.

[14] A. Chaigne, "On the use of finite differences for musical synthesis. Application to plucked stringed instruments," Journal d'Acoustique, vol. 5, no. 2, pp. 181-211, 1992.

[15] M. Karjalainen, "1-D digital waveguide modeling for improved sound synthesis," in Proc. IEEE International Conference on Acoustics, Speech and Signal Processing, vol. 2, pp. 1869-1872, Orlando, Fla, USA, May 2002.

[16] C. Erkut and M. Karjalainen, "Virtual strings based on a 1-D FDTD waveguide model: Stability, losses, and traveling waves," in Proc. Audio Engineering Society 22nd International Conference on Virtual, Synthetic and Entertainment Audio, pp. 317-323, Espoo, Finland, June 2002.

[17] C. Erkut and M. Karjalainen, "Finite difference method vs. digital waveguide method in string instrument modeling and synthesis," in Proc. International Symposium on Musical Acoustics, Mexico City, Mexico, December 2002.

[18] M. Karjalainen, C. Erkut, and L. Savioja, "Compilation of unified physical models for efficient sound synthesis," in Proc. IEEE International Conference on Acoustics, Speech and Signal Processing, vol. 5, pp. 433-436, Hong Kong, China, April 2003.

[19] A. Fettweis, "Wave digital filters: Theory and practice," Proc. IEEE, vol. 74, no. 2, pp. 270-327, 1986.

[20] M. Karjalainen, "BlockCompiler: Efficient simulation of acoustic and audio systems," in Proc. 114th Audio Engineering Society Convention, Amsterdam, Netherlands, March 2003, preprint 5756.

[21] M. Karjalainen, "Time-domain physical modeling and realtime synthesis using mixed modeling paradigms," in Proc. Stockholm Music Acoustics Conference, vol. 1, pp. 393-396, Stockholm, Sweden, August 2003.

[22] T. I. Laakso, V. Välimäki, M. Karjalainen, and U. K. Laine, "Splitting the unit delay-tools for fractional delay filter design,” IEEE Signal Processing Magazine, vol. 13, no. 1, pp. 3060, 1996.

[23] J. R. Pierce and S. A. Van Duyne, "A passive nonlinear digital filter design which facilitates physics-based sound synthesis of highly nonlinear musical instruments," Journal of the Acoustical Society of America, vol. 101, no. 2, pp. 1120-1126, 1997.

[24] R. Msallam, S. Dequidt, S. Tassart, and R. Caussé, "Physical model of the trombone including nonlinear propagation effects," in Proc. International Symposium on Musical Acoustics, vol. 2, pp. 419-424, Edinburgh, Scotland, UK, August 1997. 
[25] T. Ilmonen, "Mustajuuri-an application and toolkit for interactive audio processing," in Proc. International Conference on Auditory Display, pp. 284-285, Espoo, Finland, July 2001.

[26] M. Puckette, "Pure data," in Proc. International Computer Music Conference, pp. 224-227, Thessaloniki, Greece, September 1997.

[27] J. E. Brittain, “Thevenin's theorem,” IEEE Spectrum, vol. 27, no. 3, pp. 42, 1990.

Matti Karjalainen was born in Hankasalmi, Finland, in 1946. He received the M.S. and the Dr.Tech. degrees in electrical engineering from the Tampere University of Technology, in 1970 and 1978, respectively. Since 1980 he has been a professor in acoustics and audio signal processing at the Helsinki University of Technology in the Faculty of Electrical Engineering. In audio technology, his interest is in audio signal processing

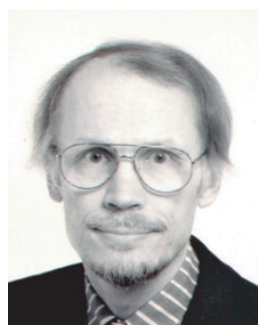
such as digital signal processing (DSP) for sound reproduction, perceptually based signal processing, as well as music DSP and sound synthesis. In addition to audio DSP, his research activities cover speech synthesis, analysis, and recognition, perceptual auditory modeling and spatial hearing, DSP hardware, software, and programming environments, as well as various branches of acoustics, including musical acoustics and modeling of musical instruments. He has written more than 300 scientific and engineering articles and contributed to organizing several conferences and workshops. Professor Karjalainen is Audio Engineering Society (AES) Fellow and Member in Institute of Electrical and Electronics Engineers (IEEE), Acoustical Society of America (ASA), European Acoustics Association (EAA), International Computer Music Association (ICMA), European Speech Communication Association (ESCA), and several Finnish scientific and engineering societies.

Cumhur Erkut was born in Istanbul, Turkey, in 1969. He received his B.S. and his M.S. degrees in electronics and communication engineering from the Yildiz Technical University, Istanbul, Turkey, in 1994 and 1997, respectively, and the Dr.Tech. degree in electrical engineering from the Helsinki University of Technology (HUT), Espoo, Finland, in 2002. Between 1998 and 2002, he worked as a researcher at the Laboratory

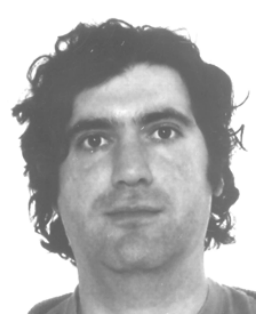
of Acoustics and Audio Signal Processing of the HUT. He is currently a postdoctoral researcher in the same institution, where he contributes to the EU-funded research project "Algorithms for the Modelling of Acoustic Interactions" (ALMA, IST-2001-33059). His primary research interests are model-based sound synthesis and musical acoustics. 